\title{
Feed-forward neural network assisted by discriminant analysis for the spectroscopic discriminantion of cracked spores Ganoderma lucidum: A prospective biotechnology production tool
}

\author{
Chee Wei Lim ${ }^{1 *}$, Sheot Harn Chan ${ }^{1}$ and Angelo Visconti ${ }^{2}$
}

\begin{abstract}
A major problem for manufacturers of cracked spores Ganoderma lucidum, a traditional functional food/Chinese medicine (TCM), is to ensure that raw materials are consistent as received from the producer. To address this, a feed-forward artificial neural network (ANN) method assisted by linear discriminant analysis (LDA) and principal component analysis (PCA) was developed for the spectroscopic discrimination of cracked spores of Ganoderma lucidum from uncracked spores. 120 samples comprising cracked spores, uncracked spores and concentrate of Ganoderma lucidum were analyzed. Differences in the absorption spectra located at $v 1\left(1143-1037 \mathrm{~cm}^{-1}\right), v 2(1660$ - $\left.1560 \mathrm{~cm}^{-1}\right), v 3\left(1745-1716 \mathrm{~cm}^{-1}\right)$ and $v 4\left(2845-2798 \mathrm{~cm}^{-1}\right)$ were identified by applying fourier transform infrared (FTIR) spectroscopy and used as variables for discriminant analysis. The utilization of spectra frequencies offered maximum chemical information provided by the absorption spectra. Uncracked spores gave rise to characteristic spectrum that permitted discrimination from its cracked physical state. Parallel application of variables derived from unsupervised LDA/PCA provided useful (feed-forward) information to achieve 100\% classification integrity objective in ANN. 100\% model validation was obtained by utilizing 30 independent samples. $v 1$ was used to construct the matrix-matched calibration curve $(n=10)$ based on 4 levels of concentration $(20 \%, 40 \%, 60 \%$ and $80 \%$ uncracked spores in cracked spores). A coefficient of correlation ( $r$ ) of 0.97 was obtained. Relative standard deviation (RSD) of $11 \%$ was achieved using $100 \%$ uncracked spores $(n=30)$. These results demonstrate the feasibility of utilizing a combination of spectroscopy and prospective statistical tools to perform non destructive food quality assessment in a high throughput environment.
\end{abstract}

Keywords: Adulteration, Artificial neural network, Cracked spores, Feed-forward, Ganoderma, Spectroscopy

\section{Introduction}

Ganoderma lucidum, a fungus commonly known as Lingzhi, has been used as a traditional functional food/ medicine for centuries by rulers of the Chinese and Japanese dynasties to achieve enhanced vitality and longevity. These formulae take on exotic forms of special tea and mushroom concoction suitable for daily intake as supplements. Owing to the perceived benefits

\footnotetext{
* Correspondence: jeff_lim@hsa.gov.sg

'Food Safety Laboratory, Applied Sciences Group, Health Sciences Authority,

11 Outram Road, 169078, Singapore

Full list of author information is available at the end of the article
}

of these highly desirable medicinal properties localized within the spores (Lin 2001) and further amplified by profit focused producers, commercial demand for Ganoderma lucidum outstripped its natural occurrence in nature. Consequently, cultivation techniques were developed to cater for mass production. Some channels used to perform cultivation include horizontal stirred tank reactor and solid state fermentation (Habijanic and Berovic, 2000; Yang et al. 2003; Hsieh and Yang 2004). Both types of cultivation strategies have been reported to yield reasonable fruit bodies suitable for general use. A major problem for manufacturers of cracked spores 
Ganoderma lucidum therefore, is to ensure the raw materials supplied are consistent (Li et al. 2011). According to Recital 11 of the European Union Regulation on the hygience of foodstuffs No 852/2004, the application of hazard analysis and critical control point (HACCP) principles to primary produce is not yet generally feasible (Cerf and Donnat, 2011). By this same principle, rapid methods (practicable in a factory environment) are therefore required to test materials prior to its conversion into the finished product.

The chemical structure of triterpenoids of Ganoderma lucidum comprised mainly of ganodermic acid and its alcohol moieties and aldehydes (Sanodiya et al. 2009), among others. Conventional analytical methods applied to characterize these triterpenoids involves the use of liquid chromatography such as reverse-phased high-performance liquid chromatography (HPLC) to separate the complex mixtures and identify them based on their absorbance at $235 \mathrm{~nm}, 243 \mathrm{~nm}$ and $251 \mathrm{~nm}$ in methanol using ultra-violet (UV) detector (Chyr and Shiao 1991; Shiao et al. 1989). Post column UV detection at $243 \mathrm{~nm}$ was then applied to quantify the triterpenoids. HPLC analysis therefore involves the administration of a series of indirect and irreversible destructive protocols. Common to all industrial food processes, the ability to obtain real-time information via an integrated and non destructive quality control system is an attractive option financially, since process delinquencies due to poor materials control may now be greatly reduced via an intermediate quality assessment step implemented at the raw materials level.

For this purpose, we developed a workflow involving the direct application of rapid FTIR and its feed-forward ANN model to perform classification of cracked spores of Ganoderma lucidum originating from a single producer to assess its raw materials (quality) consistency. Cracked spores, uncracked spores and concentrate of Ganoderma lucidum were used to construct the model by utilizing their principal frequency bands. PCA and LDA were applied on a 120 sample data pool. The values derived by applying PCA/LDA analyses were then fed into an ANN model constructed using 4 hidden nodes. Model validation was performed using 33\% (random) data set.

\section{Material and methods Samples}

120 samples comprised mainly of cultivated Ganoderma lucidum strain were analysed. These samples were received in bulk unpackaged powder form. To achieve model integrity, 90 samples comprising reference materials of cracked spores, uncracked spores and concentrate of Ganoderma lucidum were used as markers in our model building. To ensure sample uniformity, each sample matrix was homogenized using mortar and pestle prior to performing FTIR analysis.

\section{FTIR}

Spectral measurements were carried out on a Shimadzu FTIR 8400 S system equipped with a germanium coated $\mathrm{KBr}$ beam splitter, a Michelson type ( $30^{\circ}$ incident angle) interferometer and a temperature controlled high sensitivity detector (DLATGS). Spectra were recorded under diffuse reflectance mode at a resolution of $4 \mathrm{~cm}^{-1}$ set to 128 scans. Spectra were acquired and processed using the as-supplied IRSolution program for microsoft windows. Absorbance peaks were then converted to second derivative using 15 convolution points (Munakata 1998). Calculation was performed by dividing the area of the absorption band of interest with a fixed frequency band located between $810-786 \mathrm{~cm}^{-1}$. This band was represented to be a silica-related absorption band (Bosch Reig et al. 2002) originating from the sample holder used to perform spectrum acquisition. In this paper, we considered using the area (relative) of each spectrum at pre-defined frequency bands to perform model construction. These frequency bands were represented by v1 $\left(1143-1037 \mathrm{~cm}^{-1}\right), v 2\left(1660-1560 \mathrm{~cm}^{-1}\right), v 3(1745$ $\left.1716 \mathrm{~cm}^{-1}\right)$ and $v 4\left(2845-2798 \mathrm{~cm}^{-1}\right)$ respectively.

\section{Statistics and data processing}

\section{Principal component analysis (PCA)}

A commercially available Windows version of the JMP 9.0 (division of SAS Institute Inc, Cary, North Carolina, USA) was used to perform PCA, LDA and ANN analyses. For PCA analysis, the most prominent directions of the high-dimension data were identified. The dimensionality of the dataset was reduced by first applying a linear combination of the standardized original variables possessing the greatest possible variance, thereby creating the first principal component (PC 1). The second component (PC 2) was created based on the linear combination of the standardized original variables having the greatest possible variance and was uncorrelated with all previous defined components. By this principle, a reduced set of variables was achieved (Meloun et al. 1992). In this paper, unsupervised PCA was performed.

Linear discriminant analysis (LDA)

In DA, the classification variable is fixed and predicted by the continuous variables. JMP's implementation permits three types of DA(s) defined by linear, quadratic and mixed linear/quadratic. Briefly, LDA requires the $Y$ variables to be normally distributed with the same variance and covariance but with different means for each group, while quadratic discriminate analysis requires the covariance to be different across the groups. In this paper, we considered applying LDA. To assess the model's classification robustness, 30 independent samples 
comprising cracked spores Ganoderma lucidum were used to perform cross-validation.

\section{Artificial neural network (ANN)}

ANN analysis was used to assess the classification integrity predicted by the discriminant function, as well as to predict the amount of uncracked/cracked spores content by comparison with a known. Briefly, the TanH activation function was applied using one hidden layer. Values derived by applying PCA/LDA were fed into a hyperbolic tangent function $\left(\frac{e^{2 x}-1}{e^{2 x}+1}\right)$ that transforms values between -1 and 1 . It is the centred and scaled version of the logistic function, with $x$ representing the linear combination of the $\mathrm{X}$ variables. No penalty constraint was applied to the method. To predict the approximate crack/uncracked spores content (qualitative assessment) present in a known material, 4 levels of concentration of $20 \%, 40 \%, 60 \%$ and $80 \%$ uncracked spores in cracked spores were prepared.

\section{Results}

Differences in absorption spectra of Ganoderma lucidum samples

The absorption spectra of the homogenized samples were characterized by feature-rich frequency bands representative of major components of Ganoderma lucidum. These frequency bands are collectively defined by the fingerprints of polysaccharide, polysaccharide-peptide complex, $\beta$-glucans, lectins, organic germanium, adenosine, triterpenoids and nucleotides combined (Gao et al. 2004; Mizuno 1995; Liu 1999). Area-normalized spectra of cracked spores, uncracked spores and concentrate of Ganoderma lucidum are shown in Figure 1a, b and $1 \mathrm{c}$, respectively. In particular, spectral variations were observed in the region $1050-1800 \mathrm{~cm}^{-1}$ (hetero-oxy and carbonyl containing) and $2700-3000 \mathrm{~cm}^{-1}$ (aliphatic compounds) across all 3 types of reference materials. By using an $F$-test on the ratio of the variances in each dataset (Kemsleyet al. 1994), the null hypothesis that no spectral variation is caused by plant species can be rejected at the $0.10 \%$ level. This suggested that spectral variation between cracked spores, uncracked spores and concentrate of Ganoderma lucidum, is significant.

\section{Principal component analysis (PCA), linear discriminant analysis (LDA)}

In order to classify and quantify these variations, linear transformation method of PCA was first applied. 97.6\% of the variance of the original dataset was explained by the first two PCs, as shown in the 2-D score plots of PCA results in Figure 2. Frequency band $v 1$ has the highest weight on the first PC (explaining $81.0 \%$ of the variability) while $v 2, v 3$ and $v 4$ dominated the second
PC (explaining $16.6 \%$ of the variability). From Figure 2, 3 distinct clusters representing cracked spores, uncracked spore and concentrate of Ganoderma luci$d u m$ were obtained, each achieving $100 \%$ classification objective. The results suggested that a classification rule based on nearness to group means is appropriate. LDA was then applied and validation performed using 30 independent samples containing pure cracked spores. A summary of the predicted group membership of Ganoderma samples is shown in Table 1. From Table 1, it is clear that all 30 samples were classified correctly under the category of cracked spores.

\section{Artificial neural network (ANN)}

Coefficient of correlation $(r)$ value of 1.0 (both training and validation sets) was achieved for frequency bands $v 1, v 2, v 3$ and $v 4$ suggesting perfect model fit. Similarly, a root mean square error (RMSE) of $<0.1 \%$ was reported. Clearly, the results reported by the ANN model confirmed the classification outcome obtained by applying PCA/LDA analyses.

\section{Discussion}

While it is possible to improve the first PC score further by reducing the variables (frequency bands), such approach raised some concerns within the framework of spectroscopy. Indeed, while PCA and LDA are useful tools suitably used to extract features that are focused on discriminating between classes via dimension reduction strategy, the error increment due to dimension reduction has to be without sacrificing the discriminative power of classifiers (Benediktsson and Sveinsson 1997). In this work, we did not observe such limitation. Rather, by shrinking the variables pool further, the advantage of utilizing the maximum chemical information provided by the absorption spectra will not be fully tapped. For this purpose, the values obtained for the PCs and canonical functions (LDA) were fed into an ANN model using 4 hidden nodes (33\% random data holdback) to ascertain the classification outcome obtained when PCA and LDA were applied.

Within the framework of linear discriminant function, 3 distinct clusters representing cracked spores, uncracked spores and concentrate of Ganoderma lucidum were established. In order to better translate the materials quality consistency into quantifiable numbers suitable for routine monitoring purpose, a calibration curve comprising 4 levels of concentration $(n=10)$ at $20 \%$ concentration interval of cracked spores (in uncracked spores medium) was prepared. Amongst the 4 frequency bands $(v 1, v 2, v 3$ and $v 4)$ previously identified for discriminant analyses, only frequency band $v 1$ provided an acceptable $r$ value of 0.97 . This observation 


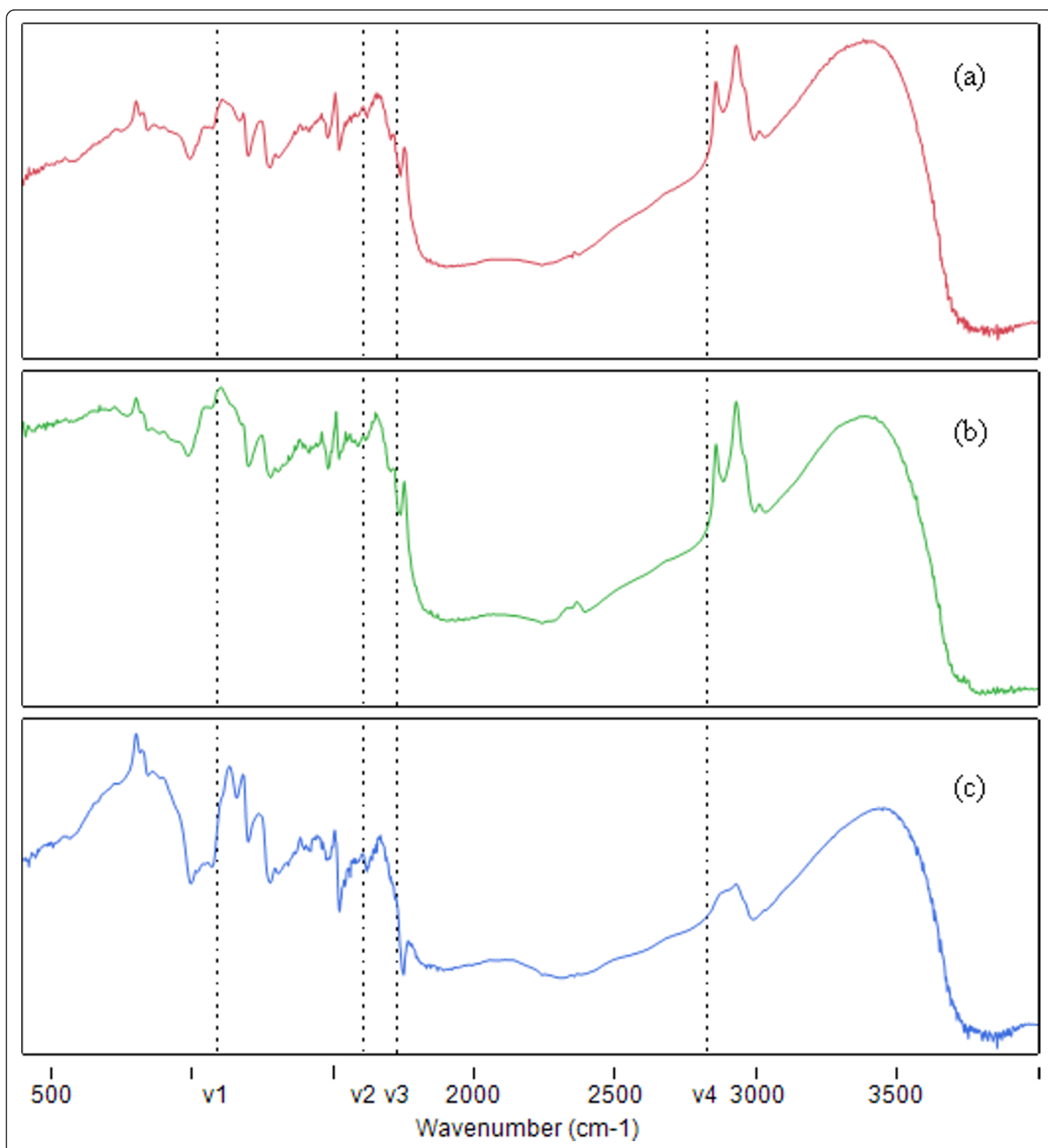

Figure 1 Area-normalised FTIR absorption spectra of a uncracked spores, b cracked spores and c concentrate of Ganoderma lucidum $v 1, v 2, v 3$ and $v 4$ represent the midband frequency of the principal frequency band used to perform PCA/LDA/ANN analysis.

is in parallel to the $v 1$ dominated first principal component score prior discussed. Using the uncracked spores as a known concentration measurement criteria ( $n=$ 30 ), a mean value of $97 \%$ (uncracked spores) was reported based on the calibration curve with a RSD value of about $11 \%$. Using the inverse correlation of cracked spores and uncracked spores, a sample categorized as pure cracked spores would therefore have a corrected composition of about $97 \pm 11 \%$ cracked spores content. By this preposition, it is possible to translate spectra into qualifiers that can be considered for routine analysis to achieve materials quality consistency 


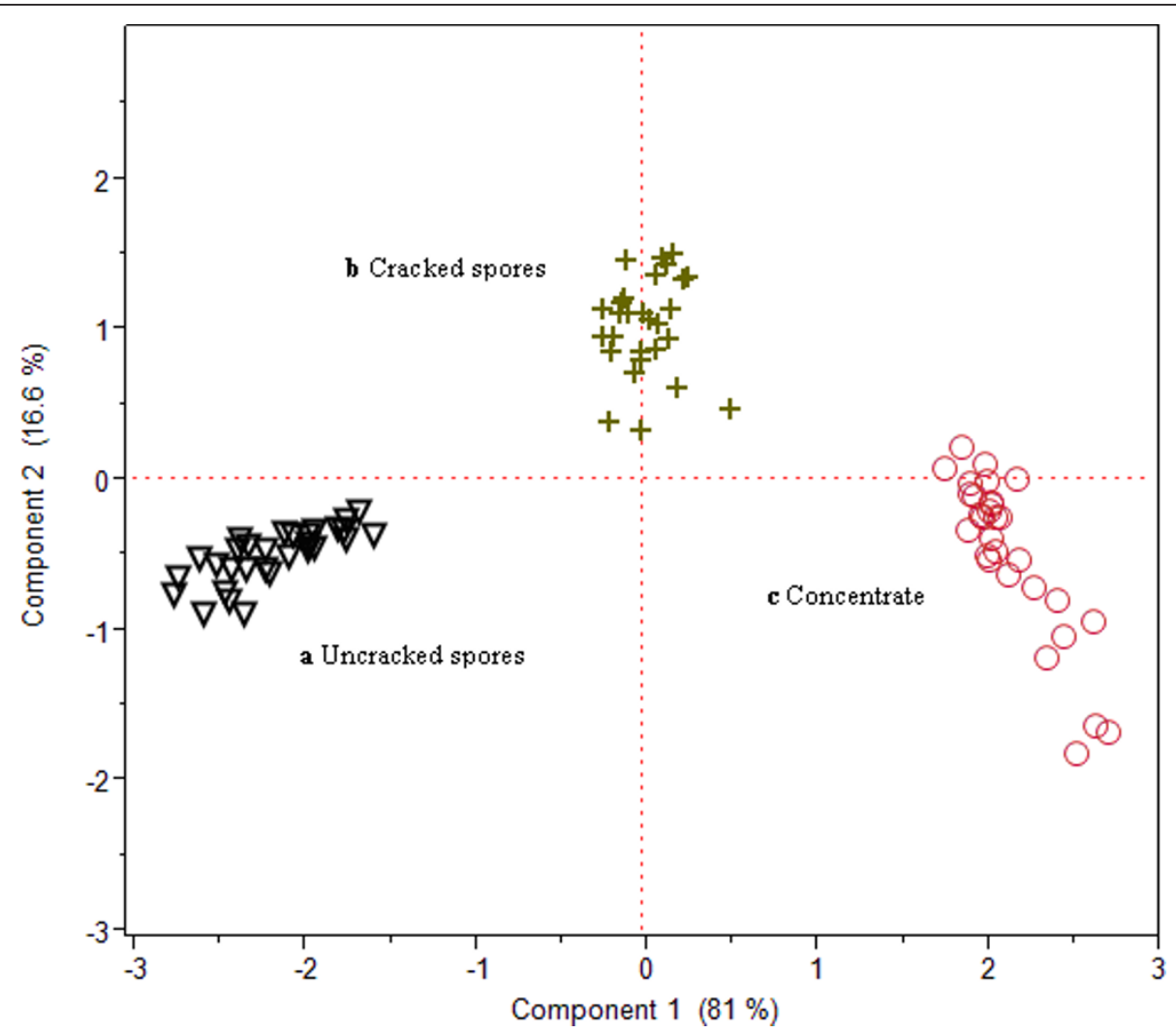

Figure 2 2-D score plots of PCA results for a uncracked spores b cracked spores and c concentrate of Ganoderma lucidum by the first two principal components.

monitoring objective (only). While the marriage of PCA/ LDA and feed-forward ANN strategies offered potential value to achieve discreet plant-based sample analyses objective, it is also important to consider expanding the model to address disproportionate variance-covariance matrices (Kemsley et al. 1994) of data sets when one cluster became enlarged (on the basis of a known single source supplier).

In brief, this work has examined the use of applying feed-forward ANN assisted by PCA/LDA analyses to discriminate cracked spores, uncracked spores and concentrate of Ganoderma lucidum to achieve materials

Table 1 Classification of uncracked spores (US), cracked spores (CS) and concentrate (C) of Ganoderma lucidum, and percentage of observations correctly classified.

\begin{tabular}{|c|c|c|c|c|c|c|c|}
\hline & & & \multicolumn{5}{|c|}{ Predicted group membership } \\
\hline & & & US & CS & C & CS (Validation) & Total \\
\hline \multirow[t]{5}{*}{ Original } & Count & US & 30 & 0 & 0 & 0 & 30 \\
\hline & & CS & 0 & 30 & 0 & 0 & 30 \\
\hline & & C & 0 & 0 & 30 & 0 & 30 \\
\hline & & CS (Validation) & 0 & 0 & 0 & 0 & 0 \\
\hline & $\%$ & & 100.0 & 100.0 & 100.0 & 0 & $100.0^{\mathrm{b}}$ \\
\hline \multirow[t]{5}{*}{ Cross-validated } & Count & US & 30 & 0 & 0 & 0 & 30 \\
\hline & & CS & 0 & 30 & 0 & 0 & 30 \\
\hline & & C & 0 & 0 & 30 & 0 & 30 \\
\hline & & CS (Validation) & 0 & 0 & 0 & $30^{\mathrm{a}}$ & 30 \\
\hline & $\%$ & & 100.0 & 100.0 & 100.0 & 100 & $100.0^{c}$ \\
\hline
\end{tabular}

a. 30 independent samples of cracked spores of Ganoderma lucidum were used to perform validation only.

b. $100.0 \%$ of original grouped cases were correctly classified.

c. $100.0 \%$ of cross-validated grouped cases were correctly classified. 
quality consistency monitoring objective. 100\% classification integrity was achieved. We found that uncracked spores contained distinctive absorption spectre that can be separated using classical FTIR and its discriminant analysis combined. These results demonstrate the feasibility of utilizing a combination of spectroscopy and prospective statistical tools to perform non destructive food quality assessment in a high throughput environment.

On hindsight, the successful marriage of spectroscopy and its statistical model perhaps lend light to the underregulated functional food/TCM industry and its processes, towards achieving quality materials supply/control and quality products suitable for a safer public consumption objectives.

\section{Acknowledgements}

The materials used in this work were sponsored by Global Active Singapore.

\section{Author details}

${ }^{1}$ Food Safety Laboratory, Applied Sciences Group, Health Sciences Authority, 11 Outram Road, 169078, Singapore ${ }^{2}$ Institute of Sciences of Food

Production, National Research Council, Via Amendola, 122/O, 70126 Bari, Italy

\section{Completing interests}

The authors declare that they have no competing interests.

Received: 3 October 2011 Accepted: 15 November 2011

Published: 15 November 2011

\section{References}

Benediktsson JA, Sveinsson JR (1997) Feature extraction for multisource data classification with artificial neural networks. International Journal of Remote Sensing, 18(4):727-740. doi:10.1080/014311697218728.

Bosch Reig F, Gimeno Adelantado JV, Moya Moreno MCM (2002) FTIR quantitative analysis of calcium carbonate (calcite) and silica (quartz) mixtures using the constant ratio method. Application to geological samples. Talanta 58:811-821

Cerf O, Donnat E (2011) Application of hazard analysis -Critical control point (HACCP) principles to primary production: What is feasible and desirable? Food Control 22:1839-1843. doi:10.1016/j.foodcont.2011.04.023.

Chyr R, Shiao MS (1991) Liquid chromatographic characterization of the triterpenoid patterns in Ganoderma lucidum and related species. Journal of Chromatography A 542:327-336

Gao Y, Lan J, Dai X, Ye J, Zhou SH (2004) A phase I//ll study of Lingzhi mushroom Ganoderma lucidum. (W. Curt.: Fr.) Lloyd (Aphyllophoromycetideae) extract in patients with type II diabetes mellitus. International Journal for Medicinal Mushrooms 6(1):33-39. doi:10.1615/ IntJMedMushr.v6.i1.30.

Habijanic J, Berovic M (2000) The relevance of solid-state substrate moisturizing on Ganoderma lucidum biomass cultivation. Food Technology and Biotechnology 38:225-228

Hsieh C, Yang FC (2004) Reusing soy residue for the solid-state fermentation of Ganoderma lucidum. Bioresource Technology 91:105-109. doi:10.1016/509608524(03)00157-3.

Kemsley EK, Belton PS, McCann MC, Ttofis S, Wilson RH, Delgadillo (1994) Spectroscopic method for the authentication of vegetable matter. Food Control 5(4):241-243. doi:10.1016/0956-7135(94)90023-X.

Lin ZB (2001) Modern Research of Ganoderma. 2nd Edn. (Beijing Medical University Press: Beijing)

Li A, Yat YW, Yap WK, Lim CW, Chan SH (2011) Discriminating authentic Nostoc flagelliforme from its counterfeits by applying alternative ED-XRF and FTIR techniques. Food Chemistry 129(2):528-532. doi:10.1016/j. foodchem.2011.01.054.
Liu GT (1999) Recent advances in research of pharmacology and clinical applications of Ganoderma P. Larst species (Aphyl- lophoromycetideae) in China. International Journal for Medicinal Mushrooms 1(1):63-68

Meloun M, Militky J, Forina M (1992) Chemometrics for analytical chemistry (pp. 224). New York: Ellis Harwood

Mizuno T (1995) Ganoderma lucidum and Ganoderma Tsugae: bioactive substances and medicinal effects. Food Review International 11(1):151-166. doi:10.1080/87559129509541025.

Munakata T (1998) Fundamentals of the New Artificial Intelligence: Beyond traditional Paradigms. Springer, New York

Sanodiya BS, Thakur GS, Baghel RK, Prasad GBKS, Bisen PS (2009) Ganoderma lucidum: A potent pharmacological macrofungus. Current Pharmaceutical. Biotechnology 10(8):717-42. doi:10.2174/138920109789978757.

Shiao MS, Lin LJ, Chen CS (1989) Determination of stero- and positional isomers of oxygenated triterpenoids by reversed phase high performance liquid chromatography. Journal of Lipid Research 30:287

Yang FC, Hsieh C, Chen HM (2003) Use of stillage grain from a rice-spirit distillery in the solid state fermentation of Ganoderma lucidum. Process Biochemistry 39:21-26. doi:10.1016/50032-9592(02)00255-8.

doi:10.1186/2191-0855-1-40

Cite this article as: Lim et al:. Feed-forward neural network assisted by discriminant analysis for the spectroscopic discriminantion of cracked spores Ganoderma lucidum: A prospective biotechnology production tool. AMB Express 2011 1:40.

\section{Submit your manuscript to a SpringerOpen ${ }^{\mathcal{O}}$ journal and benefit from:}

- Convenient online submission

- Rigorous peer review

- Immediate publication on acceptance

- Open access: articles freely available online

- High visibility within the field

- Retaining the copyright to your article

Submit your next manuscript at $\gg$ springeropen.com 